\title{
Myelom: Wenn eine Stammzelltransplantation nicht möglich ist
}

\author{
Wenn Patienten mit multiplem Myelom keine Stammzelltransplantation \\ erhalten können, lohnt sich eine kontinuierliche Therapie mit Lenalidomid \\ plus niedrigdosiertem Dexamethason. Gilt dies für jedes Lebensalter \\ gleichermaßen?
}

D as multiple Myelom betrifft bevorzugt ältere Menschen. Doch ist die Gruppe der Patienten über 75 Jahre hinsichtlich Effizienz und Verträglichkeit von Wirkstoffen wie Lenalidomid noch immer wenig untersucht. Für eine aktuelle Studie wurden 1.623 Patienten mit neu diagnostiziertem multiplem Myelom rekrutiert, darunter 567 (35\%), die älter als 75 Jahre waren. Die Teilnehmer erhielten randomisiert Lenalidomid plus niedrigdosiertes Dexamethason (Rd) fortlaufend bis zum Krankheitsprogress (Rd kontinuierlich) oder Rd für 72 Wochen (18 Zyklen; Rd18) oder Melphalan, Prednison, Thalidomid (MPT; 72 Wochen). Stratifiziert wurde nach Lebensalter $(\leq 75$ vs. $>75$ Jahre), Krankheitsstadium (International Staging System Stadium I/II vs. III) und Herkunftsland. Primärer Endpunkt war das progressionsfreie Überleben (PFS). Bei den über 75-jährigen Patienten wurden häufiger ein fortgeschrittenes Krankheitsstadium und Nierenfunktionsstörungen festgestellt.

Rd kontinuierlich reduzierte im Vergleich zu MPT das Risiko für Progression oder Tod um $31 \%$ bei allen Patienten (Hazard Ratio [HR] 0,69, 95\%-Konfidenzintervall [95\%-KI] 0,59-0,80; $\mathrm{p}<0,001$ ), um $36 \%$ bei Patienten, die höchstens 75

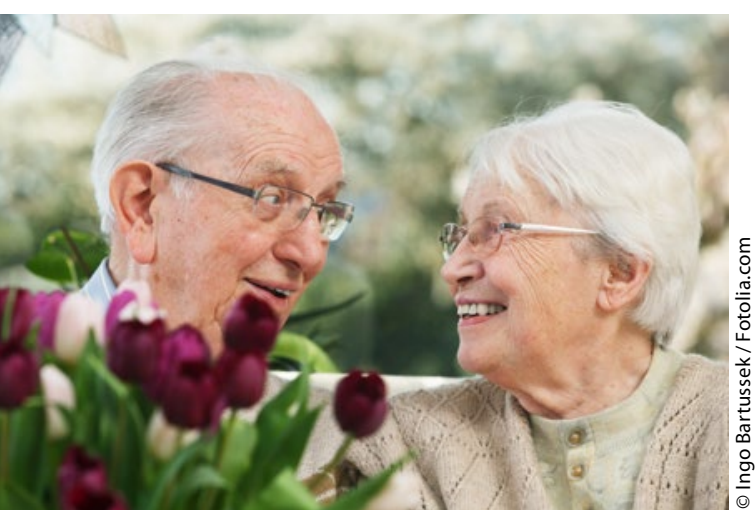

Auch ältere Patienten profitieren von Lenalidomid/Dexamethason.
Jahre alt waren (HR 0,64, $95 \%$-KI 0,53$0,77 ; \mathrm{p}<0,001)$, und um $20 \%$ bei denen, die älter als 75 Jahre waren (HR 0,80, $95 \%$-KI 0,62-1,03; $\mathrm{p}=0,084)$.

Auch das Gesamtüberleben (OS) war unter Rd kontinuierlich deutlich länger als unter MPT (alle Patienten: median 58,9 vs. 48,5 Monate; HR $0,75,95 \%$-KI 0,62-0,90). Bei den über 75-Jährigen gab es eine Differenz im medianen OS von mehr als 14 Monaten (52,3 vs. 37,8 Monate; HR 0,72, $95 \%$-KI 0,54-0,96), bei den höchstens 75 Jahre alten Patienten betrug sie rund 5 Monate (60,9 vs. 55,3 Monate; HR 0,76, $95 \%$-KI 0,60-0,96).

Unter Rd18 war das PFS demjenigen unter MPT vergleichbar. Das OS war unter Rd18 demjenigen bei kontinuierlicher $\mathrm{Rd}$ marginal unterlegen (nach 4 Jahren $3 \%$ geringer bei $\leq 75$-jährigen Patienten und $4 \%$ geringer bei $>75$-Jährigen). Akut behandlungsbedürftige Nebenwirkungen vom Grad 3/4 traten bei den beiden untersuchten Altersgruppen unter kontinuierlich Rd in gleichem Maße auf. Allerdings wurde bei den alten Patienten häufiger die Lenalidomid-Dosis reduziert.

Fazit: In der Studie bestätigte sich der Nutzen von Rd kontinuierlich bei Patienten mit neu diagnostiziertem multiplem Myelom, bei denen eine Stammzelltransplantation ausgeschlossen ist. Für jede Altersgruppe könne diese Therapie als Standard angesehen werden, so die Forscher in ihrer Bewertung.

Kathrin von Kieseritzky

Hulin C et al. Updated Outcomes and Impact of Age With Lenalidomide and Low-Dose Dexamethasone or Melphalan, Prednisone, and Thalidomide in the Randomized, Phase III FIRST Trial. J Clin Oncol. 2016 Jun 20. [Epub ahead of print].

Kommentar von Prof. Goldschmidt: In dem publizierten Update der "FIRSTStudie" fokussieren sich Cyrille Hulin und Kollegen auf das Alter der Studienpatienten. Es werden die Patientenkohorten $\leq 75$
Jahre und $>75$ Jahre analysiert. Dieser Alterscut ist bei Myelompatienten in FitnessScores gut beschrieben [Palumbo A et al. Blood. 2015;125(13):2068-74]. Hervorzuheben an dieser Arbeit sind insbesondere die prospektiven Studiendaten für ältere Myelompatienten (> 75 Jahre). Solche Daten finden sich nur selten in Publikationen zur Therapie des multiplen Myeloms.

Erfreulicherweise profitieren alle Altersgruppen von der kontinuierlichen Therapie mit Rd. Die Forscher schlussfolgern, dass Rd somit ein neuer Standard für Patienten mit multiplem Myelom ist, welche nicht mit Hochdosistherapie gefolgt von autologer Blutstammzelltransplantation behandelt werden können. Zu bedenken ist, dass heute Myelompatienten > 75 Jahre in der Regel nur mit 20 mg Dexamethason pro

\section{„In der Regel sollten ältere \\ Myelompatienten nur $20 \mathrm{mg}$ Dexamethason/Woche erhalten."}

Woche behandelt werden sollten. Auch andere Medikamente zur Therapie des multiplen Myeloms sollen im Alter > 75 Jahre reduziert angewendet werden $[\mathrm{Pa}$ lumbo A et al. Blood. 2011;118(17):4519-29]. Die Frühtoxizität bei diesen alten Patienten, z.B. durch $40 \mathrm{mg}$ Dexamethason an den Tagen 1-4, 9-12, 17-21, führte in den Untersuchungen von Vincent Rajkumar und Kollegen im ersten Jahr der Therapie zu einer signifikanten und nicht akzeptierbaren $\mathrm{Er}$ höhung der Letalität [Rajkumar SV et al. Lancet Oncol. 2010;11(1):29-37].

Zusammenfassend wird auch die Prognose von Patienten im Alter von > 75 Jahren durch die "Langzeittherapie" mit Rd verbessert. Dies ist eine wichtige Aussage zur Therapie alter Myelompatienten.

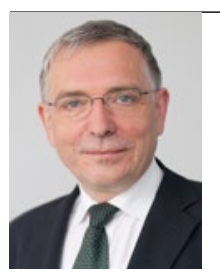

Prof. Dr. med. Hartmut Goldschmidt Sektion Multiples Myelom der Medizinischen Klinik V und des Nationalen Centrums für Tumorerkrankungen (NCT) Heidelberg hartmut.goldschmidt@ med.uni-heidelberg.de 